\title{
A model for mono- and multi-component droplet heating and evaporation and its implementation into ANSYS Fluent
}

\author{
Luke Poulton ${ }^{1}$, Oyuna Rybdylova ${ }^{1 *}$, Sergei S. Sazhin ${ }^{1}$, Cyril Crua $^{1}$, Mansour Qubeissi $^{2}$, \\ Ahmed E. Elwardany ${ }^{3}$ \\ ${ }^{1}$ Advanced Engineering Centre, School of Computing, Engineering and Mathematics, University of \\ Brighton, Brighton BN2 4GJ, UK \\ ${ }^{2}$ Centre for Mobility \& Transport, School of Mechanical, Aerospace and Automotive Engineering, \\ Coventry University, Coventry CV1 2JH, UK \\ ${ }^{3}$ Mechanical Engineering Department, Faculty of Engineering, Alexandria University, Alexandria \\ 21544, Egypt \\ *Corresponding author: O.Rybdylova@Brighton.ac.uk
}

\begin{abstract}
A model for heating and evaporation of mono- and multi-component droplets, based on analytical solutions to the heat transfer and species diffusion equations in the liquid phase, is summarised. The implementation of the model into ANSYS Fluent via User-Defined Functions (UDF) is described. The model is applied to the analysis of pure acetone, ethanol, and mixtures of acetone/ethanol droplet heating/cooling and evaporation. The predictions of the customised version of ANSYS Fluent with the newly implemented UDF model are verified against the results predicted by the previously developed in house, one-dimensional code.
\end{abstract}

Keywords: Droplets, multi-component fuel, heating, species diffusion, evaporation.

\section{Introduction}

The problem of modelling droplet heating and evaporation is a longstanding one and has been widely discussed in the literature [1-3]. Modelling of these processes is an integral part of the analysis of many engineering processes, including those which take place during spray combustion in Diesel engines [4]. The models incorporated in Computational Fluid Dynamics (CFD) codes used for the analysis of these processes, are based on a number of assumptions, the applicability of which to practical engineering problems is not at first evident [24]. One of these assumptions is that there is no temperature gradient and/or recirculation inside droplets, which could be justified in the case when liquid thermal conductivity is infinitely large. Liquid thermal conductivity is indeed much larger than that of gas in most cases, and this has been generally considered as a justification of the abovementioned assumption [4]. This approach, however, overlooks the fact that heating and evaporation of droplets in most engineering applications is not a steady state, but transient process, for which heat transfer is characterised by thermal diffusivity rather than thermal conductivity. The thermal diffusivity of liquid, in contrast to thermal conductivity, is much lower than that of gas in most cases. This allows us to question the widely used assumption that temperature gradients inside droplets can be ignored when modelling droplet heating and evaporation. The need to take into account temperature gradient inside droplets was highlighted in many experiments including those discussed in [5] (see also [2]).

The authors of [6] were perhaps the first to describe the preliminary results of implementation of a model for droplet heating and evaporation, taking into account the effects temperature gradient and recirculation inside droplets, into the commercial CFD software ANSYS Fluent. This problem was investigated later in more details in [7]. In the latter paper, the results of the implementation of the model of mono-component droplet heating and evaporation in ANSYS Fluent, using User-Defined Functions (UDF), was described. The predictions of the customised version of ANSYS Fluent were verified against experimental measurements performed at the Combustion Research Facility, Sandia National Laboratories, Livermore, California and results predicted by in-house research code for an $n$ dodecane droplet heated and evaporated in hot air. The main limitation of the model described in [7] was that it is applicable only to mono-component droplets, while most droplets used in engineering, including automotive applications, are multi-component. For the case of multi-component droplets, the process of species diffusion inside droplets needs to be taken into account alongside the heat transfer process [2]. The characteristic times of species diffusion are generally much longer than temperature relaxation times. Thus, taking into account species diffusion inside droplets is expected to be even more important than taking into account temperature gradients.

The main focus of our paper is on the generalisation of the results reported in [7], to the case of multi-component droplets, using the results of preliminary analysis presented in [6]. In contrast to mono-component droplets, the 
modelling of multi-component droplets needs to take into account both heat and species transfer processes inside droplets. The model to be used in our analysis is based on analytical solutions to the heat transfer and species diffusion equations in the liquid phase and is described in detail in [2]. The effect of recirculation in the liquid phase is taken into account based on the Effective Thermal Conductivity (ETC)/ Effective Diffusivity (ED) model [2]. The Abramzon and Sirignano approach [8] is used for modelling the gas phase.

The main ideas of the model used in our analysis are summarised in the following section, based on [2]. Then the details of the implementation of the model into ANSYS Fluent are described. The predictions of the version of ANSYS Fluent, with the new model implemented into it, will be compared with the predictions of the previously developed one-dimensional code for the case of heating/cooling and evaporation of acetone/ethanol droplets. The choice of these droplets was based on the fact that the predictions of the one-dimensional code for them were validated against experimental data [9]. Then the main results of the paper are summarised.

\section{Basic equations and approximations}

The heat transfer inside the droplets is described by the one-dimensional heat transfer equation, assuming that all processes are spherically symmetric. The analytical solution to this equation is presented as [2]:

$T(r, t)=\frac{1}{r} \sum_{n=1}^{\infty}\left\{\left(I_{n}-\frac{R_{d} \sin \lambda_{n}}{\lambda_{n}{ }^{2}} \zeta(0)\right) \frac{\exp \left(-\kappa \lambda_{n}{ }^{2} t\right)}{b_{n}}-\frac{R_{d} \sin \lambda_{n}}{b_{n} \lambda_{n}{ }^{2}} \int_{0}^{t} \frac{d \zeta(t)}{d \tau} \exp \left(-\kappa \lambda_{n}{ }^{2}(t-\right.\right.$

$\tau)) d \tau\} \sin \left[\lambda_{n} \frac{r}{R_{d}}\right]+T_{\mathrm{eff}}(t)$

where $r$ is the distance from the droplet centre, $R_{d}$ is the droplet radius, $\lambda_{n}$ are positive solutions to the eigenvalue equation (numeration starts from the first positive root, the roots are in ascending order):

$\lambda \cos \lambda+h_{0} \sin \lambda=0$,

$b_{n}=\frac{1}{2}\left(1+\frac{h_{0}}{{h_{0}{ }^{2}+\lambda_{n}{ }^{2}}^{2}}\right), I_{n}=\int_{0}^{R_{d}} \frac{r}{R_{d}} T(r, 0) \sin \left[\lambda_{n} \frac{r}{R_{d}}\right] d r, h_{0}=\frac{h R_{d}}{k_{\mathrm{eff}}}-1, h=\frac{k_{g} \mathrm{Nu}}{2 k_{\mathrm{eff}}}$,

$T(r, 0)$ is initial temperature distribution inside the droplet,

$\kappa=\frac{k_{\mathrm{eff}}}{C_{p l} \rho_{l} R_{d}^{2}}, \zeta(t)=\frac{h T_{\mathrm{eff}}(t) R_{d}}{k_{\mathrm{eff}}}, T_{\mathrm{eff}}=T_{g}+\frac{\dot{m}_{d} L}{2 \pi R_{d} \mathrm{Nu} k_{g}}$

$c_{l}$ and $\rho_{l}$ are liquid specific heat capacity and density, respectively, $L$ is specific heat of evaporation, $h$ and Nu are convection heat transfer coefficient and Nusselt number for the gas phase, respectively, $T_{g}$ is the ambient gas temperature, $k_{g}$ is gas thermal conductivity, $\dot{m}_{d}$ is droplet evaporation rate, $k_{\text {eff }}$ is the effective conductivity of the liquid phase:

$k_{\text {eff }}=\left(1.86+0.86 \tanh \left(2.225 \lg \left(\frac{\mathrm{Pe}_{l}}{30}\right)\right)\right) k_{l}$,

$k_{l}$ is the liquid thermal conductivity, $\mathrm{Pe}_{/}=\mathrm{Re}_{/} \mathrm{Pr}$, is the liquid Peclet number.

The introduction of $k$ eff was made within the so called the Effective Thermal Conductivity (ETC) model developed to take into account the effects of droplet velocity on the average value of its surface temperature. Obviously, this model cannot adequately describe the distribution of temperature inside droplets due to recirculation triggered by their relative velocities. Assuming that the time dependence of $\zeta$ is weak (this is particularly good approximation when Equation (1) is applied to a short time step (see below)), the terms proportional to the time derivative of $\zeta$ can be considered small. This allows us to simplify Equation (1) to:

$T(r, t)=\frac{1}{r} \sum_{n=1}^{\infty}\left\{\left(I_{n}-\frac{R_{d} \sin \lambda_{n}}{\lambda_{n}^{2}} \zeta(0)\right) \frac{\exp \left(-\kappa \lambda_{n}^{2} t\right)}{b_{n}} \sin \left(\lambda_{n} \frac{r}{R_{d}}\right)\right\}+T_{\mathrm{eff}}(t)$

The droplet evaporation rate $\dot{m}_{d}$ is estimated as:

$\dot{m}_{d}=-2 \pi R_{d} D \rho_{g} \ln \left(1+B_{M}\right) S h^{*}$, 
where $D$ is the binary diffusion coefficient in the gas phase,

$S h^{*}=2+\frac{\left(1+\mathrm{Re}_{d} \mathrm{Sc}\right)^{1 / 3} \max \left(1, \mathrm{Re}_{\mathrm{d}}{ }^{0.077}\right)-1}{F\left(B_{M}\right)}, F\left(B_{M}\right)=\left(1+B_{M}\right)^{0.7} \frac{\ln \left(1+B_{M}\right)}{B_{M}}$,

$B_{M}$ is the Spalding mass transfer number, $\mathrm{Sc}$ is the Schmidt number. $h$ and $\mathrm{Nu}$ are linked by the equation:

$h=\frac{k_{g} \mathrm{Nu}}{2 R_{d}}$

$\mathrm{Nu}=\frac{\ln \left(1+\mathrm{B}_{T}\right)}{B_{T}} \mathrm{Nu}^{*} \mathrm{Nu}^{*}=2+\frac{\left(1+R e_{d} \mathrm{Pr}\right)^{1 / 3} \max \left(1, R e_{d}^{0.077}\right)-1}{F\left(B_{T}\right)}, F\left(B_{T}\right)=\left(1+B_{T}\right)^{0.7} \frac{\ln \left(1+B_{T}\right)}{B_{M}}$

$\mathrm{Nu}$ is the Nusselt number, $B_{T}$ is the Spalding heat transfer number and $\mathrm{Pr}$ is the Prandtl number for the gas phase.

In the limit of infinitely large liquid thermal conductivity, Equation (1) can be simplified to (see [2] for the details):

$c_{p l} m_{d} \frac{d T}{d t}=N u \frac{k_{g}}{2 R_{d}} A_{d}\left(T_{g}-T_{s}\right)+L \dot{m}_{d}$

Where $m_{d}, A_{d}$ and $T_{s}$ are the droplet mass, surface area and surface temperature, respectively.

The derivation of Equation (1) was based on the assumption that $h$ and $T_{g}$ are constant during the process of droplet heating and evaporation which is not satisfied in most engineering applications (e.g. the reduction of $R_{d}$ during the evaporation process would lead to an increase in $h$ ). That means that neither Equation (1) nor Equation (4) can be used directly during the whole period of droplet heating and evaporation. Both these equations, however, can be used during short time steps $\Delta t$ when the assumption of constant $h$ and $T_{g}$ is valid. In this case, the solution to these equations at the end of the time step can be used as the initial condition for following time step with updated values of all other parameters, if necessary. As in [7] our analysis is based on Equation (4).

As in the case of heat transfer equation inside droplets, we assume that species diffusion inside them is described by the one-dimensional species diffusion equation and all processes are spherically symmetric. The analytical solution to this equation for the mass fractions $Y_{i}$ is presented as [2]:

$$
\begin{aligned}
Y_{i}(r, t)=\varepsilon_{i}+ & \frac{1}{r}\left[\frac{1}{b_{y 0}} \exp \left[D_{\mathrm{eff}}\left[\frac{\lambda_{0}}{R_{d}}\right]^{2} t\right]\left(I_{i 0}+\varepsilon_{i} \frac{1}{\lambda_{0}{ }^{2}}\left(1+h_{Y 0}\right) \sinh \lambda_{0}\right) \sinh \left(\lambda_{0} \frac{r}{R_{d}}\right)+\right. \\
& \left.\sum_{n=1}^{\infty} \frac{1}{b_{Y n}} \exp \left[-D_{\mathrm{eff}}\left(\frac{\lambda_{n}}{R_{d}}\right)^{2} t\right]\left(I_{i n}-\varepsilon_{i} \frac{1}{\lambda_{n}{ }^{2}}\left(1+h_{Y 0}\right) \sin \lambda_{n}\right) \sin \left(\lambda_{n} \frac{r}{R_{d}}\right)\right]
\end{aligned}
$$

where $\lambda_{0}$ and $\lambda_{n}$ are solutions to the eigenvalue equations:

$\lambda_{0} \cosh \lambda_{0}+h_{Y 0} \sinh \lambda_{0}=0, \lambda_{n} \cos \lambda_{n}+h_{Y n} \sin \lambda_{n}=0,(n=1,2, \ldots)$

$\lambda_{n}(n \geq 1)$ are in ascending order, $\varepsilon_{i}=\frac{Y_{v i s}}{\sum_{i} Y_{v i s}}, Y_{v i s}$ is vapour species mass fractions at the surface of the droplet,

$$
\begin{aligned}
& b_{Y 0}=-\frac{R_{d}}{2}\left(1+\frac{h_{Y 0}}{h_{Y O}^{2}-\lambda_{0}^{2}}\right), b_{Y n}=\frac{R_{d}}{2}\left(1+\frac{h_{Y 0}}{h_{Y o}^{2}+\lambda_{n}^{2}}\right), h_{0 Y}=-\left(1+\frac{\alpha R_{d}}{D_{l}}\right), \\
& I_{i 0}=\int_{0}^{R_{d}} \frac{r}{R_{d}} Y_{i}(r, 0) \sinh \left[\lambda_{0} \frac{r}{R_{d}}\right] d r, I_{i n}=\int_{0}^{R_{d}} \frac{r}{R_{d}} Y_{i}(r, 0) \sin \left[\lambda_{n} \frac{r}{R_{d}}\right] d r, n \geq 1,
\end{aligned}
$$

$Y_{i}(r, 0)$ Is the initial ith species mass fraction distribution inside the droplet.

$D_{\text {eff }}$ is the effective species diffusivity in the liquid phase assumed to be the same for all species and defined as [2]

$D_{\mathrm{eff}}=\left(1.86+0.86 \tanh \left(2.225 \log \left(\frac{\mathrm{Pe}_{l}}{30}\right)\right)\right) D_{l}$ 
$D_{l}$ is the liquid diffusivity, $\mathrm{Pe}_{/ Y}=\mathrm{Re}_{/} \mathrm{Sc}_{/}$is the liquid Peclet number referring to species diffusion.

The introduction $D_{\text {eff }}$ was made within the so called Effective Diffusivity (ED) model developed to take into account the effects of droplet velocity on the average values of its surface mass fractions in the liquid phase. This model cannot adequately describe the distribution of species inside droplets due to recirculation triggered by their relative velocities. Vapour molar fractions of species at the surface of the droplets and the corresponding molar fractions in the liquid phase are linked by Rault law [2].

\section{Implementation of the model into ANSYS Fluent}

Equation (7) was implemented into the customised version of ANSYS Fluent alongside with the previously implemented Equation (4). The right-hand sides of these equations were calculated via the User Defined Functions (UDF); thermodynamic and transport parameters were calculated based on the average temperature inside the droplets, using Simpson's method [10]. The droplet volume was discretised into $N_{L}=500$ concentric layers to calculate the series in Equations (4) and (7). The integrals ( $I_{n}$ and $I_{\text {in }}$ ) and average temperatures inside droplets were also calculated using the Simpson's method with 501 points for 500 layers. At each time step temperatures and species distributions were calculated from Equations (4) and (7). Time-steps for calculations were taken equal to $\delta t=10^{-6}$ seconds. The roots of the eigenvalue Equations (2) and (8) were found using the bisection method with accuracy of $10^{-8}$.

\section{Results and discussion}

The newly developed customised version of ANSYS Fluent was applied to the analysis of cooling and evaporation of droplets of ethanol, acetone and mixture of ethanol and acetone, as described in the experiments the results of which are presented in [9]. The transport and thermodynamic properties of acetone and ethanol were taken from [9]. The initial droplet temperatures were in the range 293-296 K, while the temperatures of ambient air were in the range $305-312 \mathrm{~K}$ at atmospheric pressure. Gas parameters in the vicinity of the droplet surface were calculated based on reference temperature $T_{\text {ref }}=\left(2 T_{s}+T_{g}\right) / 3.200$ eigenvalues were used to calculate temperature distribution and 10 eigenvalues were used to calculate species mass fraction distribution. Gas and liquid properties are taken from $[5,11]$.

The results predicted by ANSYS Fluent were verified against the results predicted by the one-dimensional in-house code. The predictions of the latter code, in their turn, were verified against the predictions of the code based on the numerical solutions of the heat transfer and species diffusion equations [9]. This allows us to use the abovementioned code $(\mathrm{IHC})$ as the reference code to verify the results for heating and evaporation of droplets of various compositions predicted by the new customised version of ANSYS Fluent.

The results of the comparison between ANSYS Fluent and in-house code for the temperatures at the centre and surface of the droplet and droplet average temperature are shown in Figs. 1-5 for various droplet and gas parameters and various compositions of droplets: pure acetone (Fig. 1), pure ethanol (Fig. 2), 75\% ethanol and $25 \%$ acetone (Fig. 3), 50\% ethanol and 50\% acetone (Fig. 4), 25\% ethanol and 75\% acetone (Fig. 5). Input parameters used in calculations are summarised in Table 1. As can be seen from these figures, in all cases the agreements between Fluent and IHC results are reasonably good. The percentage errors for the abovementioned three temperatures after $7 \mathrm{~ms}$ is between $1.0-4.0 \%$ for acetone, 6.0-6.1\% for ethanol, between $2.0-3.5 \%$ for the mixture with $25 \%$ of ethanol, between $0.5-1.8 \%$ for the mixture with $50 \%$ of ethanol and between $0.3-1.8 \%$ for the mixture with for $75 \%$ of ethanol. Note that the results shown in Figs. 1 and 2 were obtained without using the new features of the ANSYS Fluent code developed in our work (they could be obtained based on the version of the code developed in [7]). In all cases shown in Figs. 1-5 the changes in droplet radii were small and the corresponding curves are not shown.

Table 1 Droplet and gas temperatures, droplet diameters and approximations of droplet velocities, inferred from [11] and used in calculations.

\begin{tabular}{|l|l|l|l|l|}
\hline Composition & $\begin{array}{l}\text { Droplet } \\
\text { temperature } \\
(\mathrm{K})\end{array}$ & $\begin{array}{l}\text { Droplet } \\
\text { diameter } \\
(\mu \mathrm{m})\end{array}$ & $\begin{array}{l}\text { Gas } \\
\text { temperature } \\
(\mathrm{K})\end{array}$ & $\begin{array}{l}\text { Droplet velocity } \\
\text { approximations in m/s } \\
(\mathrm{t} \text { is in ms })\end{array}$ \\
\hline acetone & 308.25 & 143.4 & 294.65 & $12.81-0.316 t$ \\
\hline ethanol & 311.15 & 140.8 & 295.15 & $12.30-0.344 t$ \\
\hline$+25 \%$ ethanol & 305.65 & 133.8 & 294.25 & $12.75-0.370 t$ \\
\hline$+50 \%$ ethanol & 310.65 & 142.7 & 293.95 & $12.71-0.488 t$ \\
\hline$+75 \%$ ethanol & 311.75 & 137.1 & 294.75 & $12.28-0.306 t$ \\
\hline
\end{tabular}


As can be seen in Figs. 1-5 the difference between the temperatures at the centre and surface of the droplets and droplet average temperatures is approximately 2-9 K, which cannot be ignored in most engineering applications. This difference was ignored in the conventional version of ANSYS Fluent (e.g. [4]), which cannot be justified in the general case. The agreement between ANSYS Fluent and IHC results gives us confidence in applying the new customised version of ANSYS Fluent to more complex problems that those presented in Figures. 1-5.

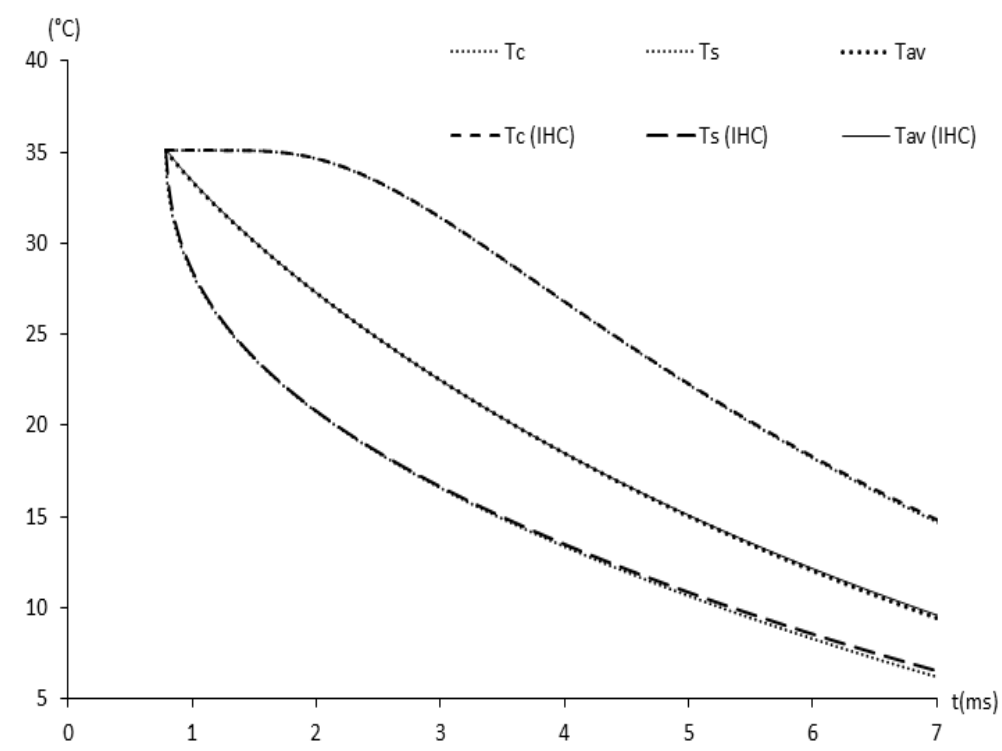

Figure 1. Time evolution of an acetone droplet surface, average and centre temperatures $\left(T_{s}, T_{a v}\right.$ and $T_{c}$ ) (see Table 1). ANSYS Fluent results (dotted curves) and compared with results of the previously developed In house code (IHC) (dashed and solid curves).

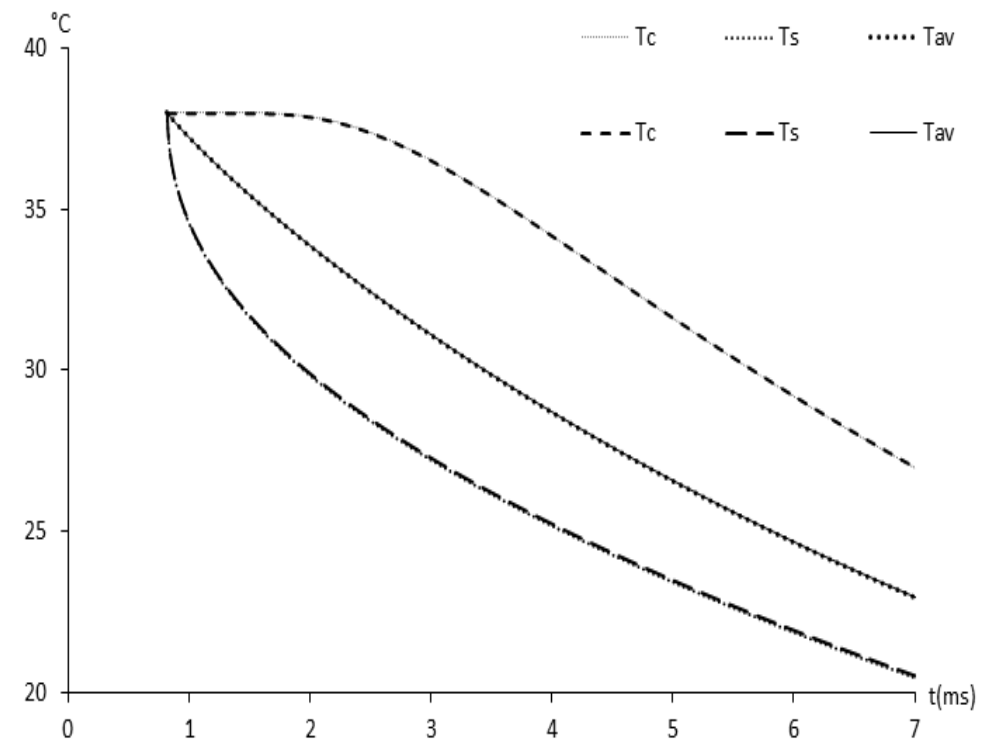

Figure 2. Time evolution of ethanol droplet surface, average and centre temperatures $\left(T_{s}, T_{a v}\right.$ and $\left.T_{c}\right)$ (see Table 1). ANSYS Fluent results (dotted curves) and compared with results of the previously developed In house code (IHC) (dashed and solid curves). 
ILASS - Europe $2017,6^{\text {th }}-8^{\text {th }}$ September, Valencia, Spain.

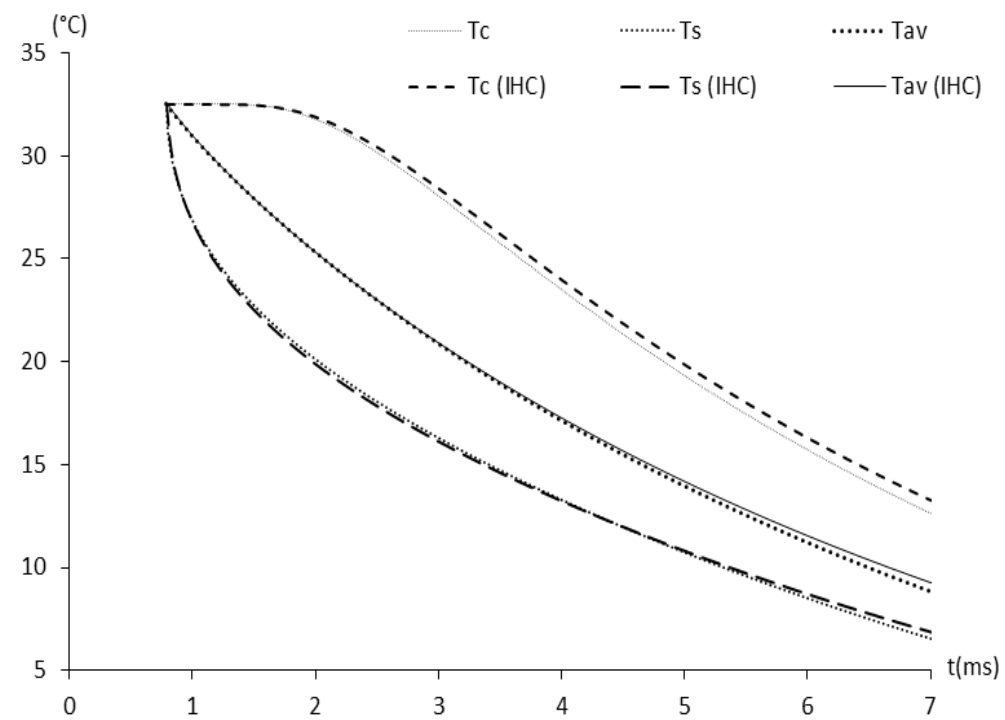

Figure 3. Time evolution of $75 \%$ ethanol and $25 \%$ acetone droplet surface, average and centre temperatures $\left(T_{s}, T_{a v}\right.$ and $T_{c}$ ) (see Table 1). ANSYS Fluent results (dotted curves) and compared with results of the previously developed In house code (IHC) (dashed and solid curves).

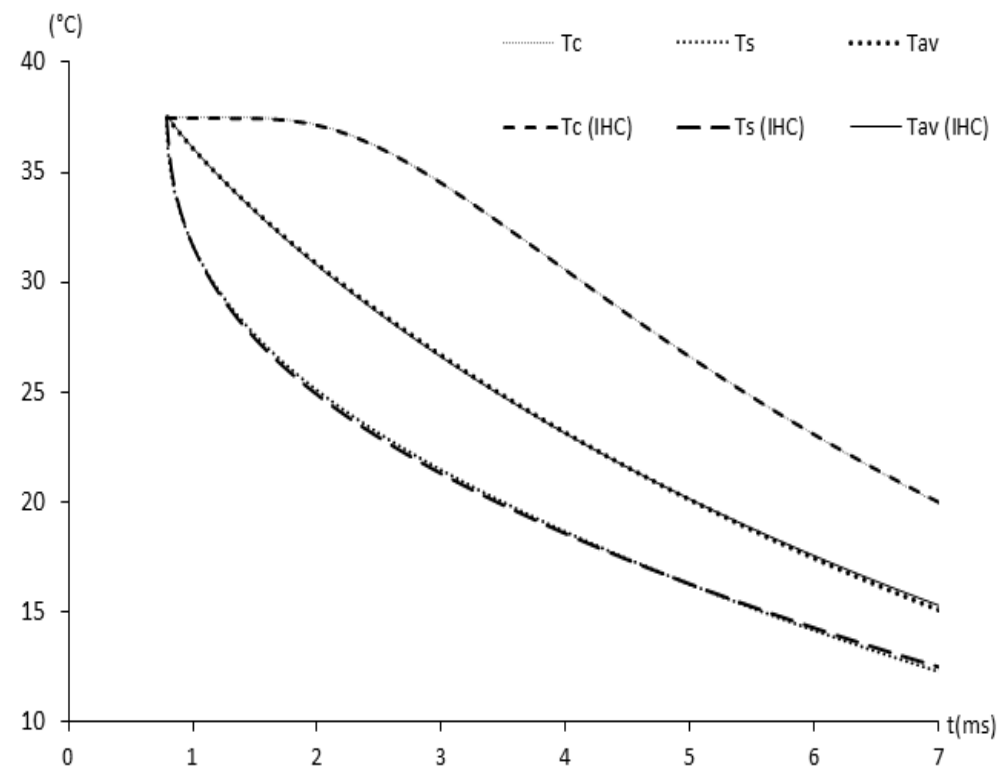

Figure 4. Time evolution of 50\% ethanol $50 \%$ acetone droplet surface, average and centre temperatures $\left(T_{s}, T_{a v}\right.$ and $T_{c}$ ) (see Table 1). ANSYS Fluent results (dotted curves) and compared with results of the previously developed In house code (IHC) (dashed and solid curves). 


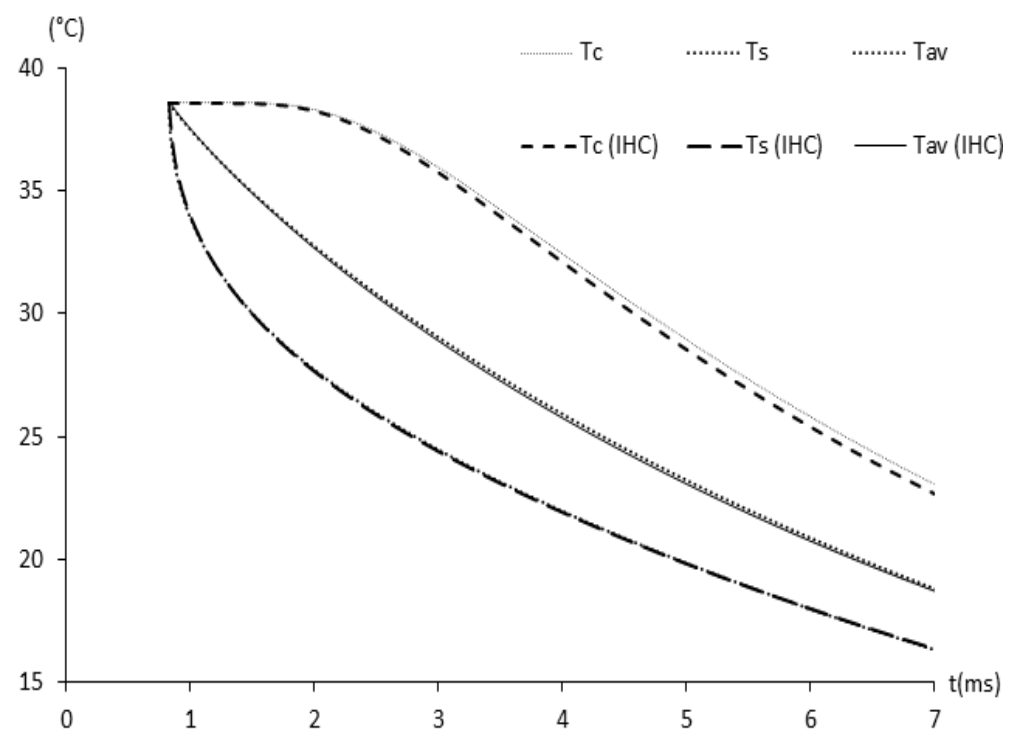

Figure 5. Time evolution of $25 \%$ ethanol $75 \%$ acetone droplet surface, average and centre temperatures ( $T_{s}, T_{a v}$ and $T_{c}$ ) (see Table 1). ANSYS Fluent results (dotted curves) and compared with results of the previously developed In house code (IHC) (dashed and solid curves).

\section{Conclusions}

A model for heating and evaporation of mono- and multi-component droplets, based on analytical solutions to the heat transfer and species diffusion equations in the liquid phase, is summarised. The implementation of the model into ANSYS Fluent via User-Defined Functions (UDF) is described. The model is applied to the analysis of pure acetone, ethanol, and mixtures of acetone/ethanol droplets heating/cooling and evaporation. The predictions of the customised version of ANSYS Fluent with the new model implemented into it are verified against the results predicted by previously developed one-dimensional in-house code based on the analytical solutions to the heat transfer and mass diffusion equations. The latter code in its turn was verified against the predictions of the in-house code developed at the University of Nancy (France) and validated against experimental data obtained at the same university.

The agreement between the predictions of these codes is shown to be reasonably good for mono-component (pure acetone and ethanol) and multi-component droplets comprising acetone with 25, 50 and 75-percentage mass fractions of ethanol, with input parameters used for pervious validation of the in-house code. This gives us confidence in using the new customised version of ANSYS Fluent for the analysis of more complex engineering processes.

\section{Acknowledgements}

The authors would like to recognise that this work was supported by the UK's Engineering and Physical Science Research Council, a studentship to support one of the authors (LP) [EPSRC grant EP/N509607/1; EP/K005758/1; EP/K020528/1; EP/M002608/1] 


\section{Nomenclature.}

\begin{tabular}{|c|c|c|c|}
\hline$B_{M} ; B_{T}$ & Spalding mass and heat transfer numbers & $\operatorname{Re}$ & Reynolds number \\
\hline$b_{n}$ & Parameter introduced in (1) & Sc & Schmidt number \\
\hline$b_{Y n}$ & Parameter introduced in (7) & Sh & Sherwood number \\
\hline$c_{p}$ & Specific heat capacity at constant pressure & $t$ & Time \\
\hline$D$ & Binary diffusivity coefficient of vapour in air & $T$ & Temperature \\
\hline$F$ & Function introduced in $\mathrm{Sh}^{\star}$ and $\mathrm{Nu}^{*}$ & $v$ & Velocity \\
\hline$h$ & Convection heat transfer coefficient & Y & Mass fraction \\
\hline$h_{0}$ & Parameter introduced in (2) & $x$ & Molar fraction \\
\hline$h_{Y O}$ & Parameter introduced in (8) & & \\
\hline $\ln$ & Integrals, used in Solutions (1) and (4) & \multicolumn{2}{|c|}{ Greek symbols } \\
\hline lin & Integrals, used in Solution (7) & $\varepsilon_{\mathrm{i}}$ & Vapour species mass fractions \\
\hline j & Parameter, defined in (3) & $\mathrm{k}$ & Parameter introduced in (1) \\
\hline$k$ & Thermal conductivity & $\lambda_{n}$ & Eigenvalues \\
\hline$L$ & Latent heat of evaporation & $\mu$ & Dynamic viscosity \\
\hline$m$ & Mass & $\rho$ & Density \\
\hline$\dot{m}_{d}$ & Evaporation rate & $\varphi$ & Parameter defined by Equation (4) \\
\hline M & Molar mass & $x$ & Correction function defined by Equation (1c) \\
\hline$N_{L}$ & Number of layers inside a droplet & $\zeta$ & parameter defined by (1c) \\
\hline $\mathrm{Nu}$ & Nusselt number & & \\
\hline $\mathrm{Pe}$ & Peclet number & \multicolumn{2}{|c|}{ Subscripts } \\
\hline$p$ & Pressure & $d$ & Droplet \\
\hline $\mathrm{Pr}$ & Prandtl number & eff & Effective \\
\hline$q$ & Heat flux & $g$ & Gas \\
\hline$r$ & Distance from the centre of the droplet & int & Internal \\
\hline$R_{d}$ & Radius of a droplet & l & Liquid \\
\hline
\end{tabular}

\section{References}

[1] Sazhin, S.S., 2006, Progress in Energy and Combustion Science 32(2), pp. 162-214.

[2] Sazhin, S.S., 2014, "Droplets and Sprays". Springer.

[3] Sazhin, S.S., 2017, Fuel, 196, pp. 69-101.

[4] Sazhina, E.M., Sazhin, S.S., Heikal, M.R., Babushok, V.I., Johns, R.J.T., 2000, Combustion Science and Technology, 160(1), pp. 317-344.

[5] Maqua, C., 2007, Contribution à la compréhension de l'évaporation de gouttes de combustible bi-composant à l'aide de méthodes optique, PhD Thèse, Nancy-Université, 2007.

[6] Elwardany, A.E., Sazhin, S.S., Castanet, G., Lemoine, F. and Heikal, M.R., 2011, Proceedings of ILASS Europe 2011, 24th European Conference on Liquid Atomization and Spray Systems, Estoril, Portugal, 5-7 September 2011, paper 1018 (CD).

[7] Rybdylova, O., Al Qubeissi, M., Braun, M., Crua, C., Manin, J., Pickett, L.M., de Sercey, G., Sazhina, E.M., Sazhin, S.S, Heikal, M., 2016, International Communications in Heat and Mass Transfer, 76, pp. 265-270.

[8] Abramzon B., Sirignano W.A., 1989, International Journal of Heat and Mass Transfer 32. pp. 1605-1618.

[9] Elwardany, A.E., Gusev, I.G., Castanet, G., Lemoine, F., Sazhin, S.S., 2011, Atomization and Sprays 21, pp. 907-931.

[10] Eagle, W.E., Musculus, M., Oefelein, J.C., Malbec, L.-M., Bruneaux, G., 2014. Measuring transient entrainment rates of a confined vaporizing diesel jet, in: 26th ILASS Americas, Portland (OR), USA, May, 2014.

[11] Sazhin, S.S., Elwardany, A., Krutitskii, P. A., Castanet, G. Lemoine, F., Sazhina, E. M., Heikal, M. R., 2010, International Journal of Heat and Mass Transfer, 53, pp. 4495-4505. 